\title{
Traditional and novel correlates of adiposity and cardiometabolic risk among young healthy adults in the North West of England
}

\author{
F. Amirabdollahian ${ }^{1}$, C.J. Macdonald-Clarke ${ }^{1}$, E.K. Lees ${ }^{1}$, T. Harrison ${ }^{2}$ and I.G. Davies ${ }^{2}$ \\ ${ }^{1}$ School of Health Sciences, Liverpool Hope University, Liverpool, L16 9JD and ${ }^{2}$ School of Sports Studies, Leisure and \\ Nutrition, Liverpool John Moores University, Liverpool L17 6BD
}

Excessive adiposity is associated with increased cardiometabolic $(\mathrm{CM})$ risk $^{(1)}$. The discriminatory power of traditional proxy indicators of adiposity such as Body Mass Index (BMI), Waist Circumference (WC) and Waist to Hip Ratio (WHpR) has been frequently challenged $^{(1,2)}$. In recent years, several novel proxy measures of adiposity such as Waist to Height Ratio $(\mathrm{WHtR})^{2}$, Clinica Universidad de Navarra - Body Adiposity Estimator (CUN-BAE) ${ }^{(3)}$ and A Body Shape Index (ABSI) ${ }^{(4)}$ and have been suggested as alternatives to the traditional measures. The aim of this study was to investigate which proxy measure of anthropometric adiposity has the strongest association with CM risk indices in healthy young adults in North West England.

After obtaining ethical approval, 396 (171 male and 225 female) participants aged 18-24 years were recruited in a cross-sectional study. Anthropometric, dietary and laboratory measures of CM risk were assessed including: percentage body fat (\%BF measured via bioelectrical impedance Tanita ${ }^{\mathrm{TM}}$ ), blood pressure (BP), 3-day validated food diary and fasting capillary whole blood glucose and lipid profile. Traditional (BMI, WC, WHpR) and novel (CUN-BAE, ABSI and WHtR) proxy indicators of adiposity were assessed or calculated using standardised techniques ${ }^{(2-4)}$. The strength of the association of these measures with CM risk indices were then compared based on the strength of the Pearson correlation coefficient in males (M) and females (F) (Table 1).

Table 1. Pearson correlation coefficient of the association between cardiometabolic risk indices and proxy indicators of adiposity $(* \mathrm{p}<0 \cdot 05, * * \mathrm{p}<0 \cdot 01)$.

\begin{tabular}{|c|c|c|c|c|c|c|c|c|c|c|c|c|}
\hline & \multicolumn{2}{|c|}{$\begin{array}{c}\text { BMI } \\
\mathrm{kg} / \mathrm{m} 2\end{array}$} & \multicolumn{2}{|c|}{$\begin{array}{l}\text { WC } \\
\mathrm{cm}\end{array}$} & \multicolumn{2}{|c|}{$\begin{array}{c}\text { WHpR } \\
\%\end{array}$} & \multicolumn{2}{|c|}{$\begin{array}{c}\text { CUNBAE } \\
\mathrm{m} 11 / 6 \mathrm{k}-2 / 3\end{array}$} & \multicolumn{2}{|c|}{ ABSI } & \multicolumn{2}{|c|}{ WHtR } \\
\hline & $\mathrm{M}$ & $\mathrm{F}$ & M & $\mathrm{F}$ & M & $\mathrm{F}$ & $\mathrm{M}$ & $\mathrm{F}$ & M & $\mathrm{F}$ & M & $\mathrm{F}$ \\
\hline \multicolumn{13}{|l|}{ Anthropometry } \\
\hline$\% \mathrm{BF}$ & $25^{* *}$ & $61^{* *}$ & $26 * *$ & $68 * *$ & $0 \cdot 06$ & 0 & $24 * *$ & $.79 * *$ & $0 \cdot 15$ & $0 \cdot 01$ & $31 * *$ & $.59 * *$ \\
\hline Systolic BP (mmHg) & 0.02 & 0.07 & 0 & $13 *$ & $-0 \cdot 11$ & 0.07 & 0.05 & $0 \cdot 12$ & $-0 \cdot 06$ & $0 \cdot 03$ & -0.02 & 0.07 \\
\hline Diastolic BP (mmHg) & $0 \cdot 04$ & $20 * *$ & $0 \cdot 06$ & $.22 *$ & $0 \cdot 13$ & $20 * *$ & $0 \cdot 04$ & $22 * *$ & 0.07 & $0 \cdot 05$ & $0 \cdot 1$ & $22 * *$ \\
\hline \multicolumn{13}{|l|}{ Dietary } \\
\hline Average energy intake (kcal/d) & $-0 \cdot 05$ & 0 & $-0 \cdot 1$ & $0 \cdot 04$ & $-0 \cdot 01$ & -0.06 & $-0 \cdot 03$ & -0.09 & $-0 \cdot 04$ & $0 \cdot 13$ & -0.08 & $0 \cdot 05$ \\
\hline Energy from fat $(\%)$ & -0.09 & $0 \cdot 11$ & $0 \cdot 06$ & $18 * *$ & 0 & 0.03 & $-0 \cdot 1$ & $0 \cdot 12$ & $\cdot 15^{*}$ & $0 \cdot 1$ & 0.02 & $16 * *$ \\
\hline Energy from saturated fat $(\%)$ & $-0 \cdot 11$ & $0 \cdot 1$ & $0 \cdot 02$ & $16 *$ & $-0 \cdot 01$ & $0 \cdot 02$ & $-0 \cdot 13$ & $0 \cdot 11$ & $0 \cdot 12$ & $0 \cdot 1$ & -0.01 & $.15 * *$ \\
\hline Energy from sugar $(\%)$ & $-0 \cdot 01$ & $-0 \cdot 1$ & $-0 \cdot 1$ & $-0 \cdot 1$ & $0 \cdot 04$ & -0.08 & $-0 \cdot 01$ & -0.07 & -0.09 & $-0 \cdot 05$ & $-0 \cdot 05$ & -0.09 \\
\hline \multicolumn{13}{|l|}{ Laboratory } \\
\hline Blood Cholesterol (mmol/L) & $0 \cdot 15$ & $0 \cdot 05$ & $0 \cdot 11$ & $0 \cdot 1$ & $-0 \cdot 01$ & 0 & $16^{*}$ & $0 \cdot 1$ & $0 \cdot 02$ & $0 \cdot 06$ & $16^{*}$ & 0.09 \\
\hline Blood TG (mmol/L) & $0 \cdot 02$ & $0 \cdot 6$ & 0.08 & $0 \cdot 06$ & 0.07 & $-0 \cdot 01$ & $0 \cdot 03$ & 0.08 & $0 \cdot 09$ & $-0 \cdot 01$ & 0.08 & $0 \cdot 06$ \\
\hline Blood LDL (mmol/L) & $0 \cdot 14$ & $0 \cdot 1$ & $0 \cdot 03$ & $0 \cdot 14$ & $-0 \cdot 01$ & $-0 \cdot 11$ & $0 \cdot 15$ & $23 * *$ & $-0 \cdot 08$ & $-0 \cdot 04$ & $0 \cdot 08$ & 0.09 \\
\hline Blood Glucose (mmol/L) & $-0 \cdot 04$ & $15^{*}$ & $0 \cdot 11$ & $19 * *$ & $.16 *$ & $.33 * *$ & $-0 \cdot 05$ & $0 \cdot 12$ & $17 *$ & $0 \cdot 12$ & 0.09 & $19 * *$ \\
\hline
\end{tabular}

For men, most novel and traditional proxy measures showed weak associations with measured \%BF. While there were occasional correlations with other dietary and laboratory correlates of CM risk, both CUN-BAE and WHtR showed weak but significant association with $\% \mathrm{BF}$ and whole blood total cholesterol. For women, CUN-BAE correlated the strongest with \%BF, while WC and WHtR demonstrated weak but (very) significant associations with various anthropometric, dietary and laboratory indices of CM risk. The findings suggest that for young adults in general, ABSI and WHpR show no or limited potential as proxy indicators of adiposity. Furthermore, the findings propose that gender specific proxy indicators may be required and, specifically for women, use of WC, WHtR and CUN-BAE may be more appropriate than BMI. This might be due to differences in adipose tissue type and distribution.

1. Haslam D, James WPT (2005) The Lancet 366, 1197-1209

2. Ashwell M, Gunn P, Gibson S (2012) Obesity Reviews 13, 275-286

3. Fuster-Parra P, Bennasar-Veny M, Tauler P et al. (2015) PLoS ONE 10, e0122291

4. Krakauer NY and Krakauer JC (2012) PLoS ONE 7, e39504 